\title{
Validación de una escala para evaluar competencias investigativas en docente de Básica y Media
}

\section{Validation of a scale to evaluate research competencies in elementary and middle school teachers in the Colombian context}

\author{
César Augusto Hernández Suárez ${ }^{1}$ \\ Audin Alioso Gamboa Suárez² \\ William Rodrigo Avendaño Castro ${ }^{3}$
}

UFPS

\section{RESUMEN}

El propósito del artículo es mostrar el diseño y validación de una escala de diagnóstico de medición para identificar las competencias en conocimientos y habilidades investigativas en los docentes de básica y media en el contexto colombiano. El desarrollo del instrumento se hizo 1 Magister en Educación Matemática por la Universidad Nacional Experimental del Táchira (Venezuela). Docente investigador de la Universidad Francisco de Paula Santander. Correo electrónico: cesaraugusto@ufps.edu.co. Orcid: 0000-0002-7974-5560.

2 Doctor en Ciencias de la Educación por la Universidad de Cartagena (Colombia). Docente investigador de la Universidad Francisco de Paula Santander. Correo electrónico: audingamboa@ufps.edu.co. Orcid: 0000-00019755-6408.

3 Doctor en Ciencias Sociales y Humanas por la Pontificia Universidad Javeriana. Docente investigador de la Universidad Francisco de Paula Santander. Correo electrónico: williamavendano@ufps.edu.co. Orcid: 0000-00027510-8222. bajo un enfoque cuantitativo, en cuatro etapas: en primer lugar, mediante una revisión de la literatura sobre el constructo, se identificaron conocimientos y habilidades de investigación y luego se definieron operativamente. Los ítems del instrumento fueron tipo Liker con cinco (5) alternativas de respuestas, 3 dimensiones (identificación y organización de información, generación y divulgación del conocimiento científico) para un total de 31 ítems. Se realizó un estudio piloto, una validación semántica y de contenido por juicio de expertos y su confiabilidad se midió utilizando el método de consistencia interna. La etapa final, consistió en evaluar las competencias investigativas en una muestra de con 32 docentes. Las correlaciones entre factores del instrumento muestran relación 
y dependencia entre ellos, indicando la validez del cuestionario.

\section{PALABRAS CLAVE:}

Competencias de investigación, profesores de educación básica, generación de conocimientos, difusión de conocimientos, juicio de expertos, construcción de escalas

\section{ABSTRACT}

The purpose of the article is to show the design and validation of a measurement diagnostic scale to identify competencies in knowledge and investigative skills in elementary and middle school teachers in the Colombian context. The instrument was developed under a quantitative approach, in four stages: first, through a review of the literature on the construct, knowledge and research skills were identified and then operationally defined. The instrument items were Liker type with five (5) alternative responses, 3 dimensions (identification and organization of information, generation and dissemination of scientific knowledge) for a total of 31 items. A pilot study, a semantic and content validation by expert judgment was carried out and its reliability was measured using the internal consistency method. The final stage consisted of evaluating the research skills in a sample of 32 teachers. The correlations between factors of the instrument show a relationship and dependence between them, indicating the validity of the questionnaire.

\section{KEYWORDS:}

Research skills, basic education teachers, knowledgegeneration, knowledge dissemination, expert judgement, scale construction.

\section{INTRODUCCIÓN}

El sistema educativo de hoy en día en consonancia con la sociedad del conocimiento exige del docente un conjunto de competencias que le faciliten el diagnóstico y resolución de los múltiples problemas que enfrentan la escuela y su entorno; todo ello, con el propósito de elevar la calidad de la educación, la participación activa de los miembros de la comunidad escolar y fomentar la calidad en las instituciones educativas, cuestión que es posible alcanzar a través de procesos investigativos que aproximen al docente a la realidad escolar y lo conduzcan a asumir su quehacer como una práctica reflexiva, participativa y colectiva, lo cual plantea como requerimiento que éste posea herramientas pedagógicas, saberes teóricos y destrezas básicas para la búsqueda de soluciones a los problemas dentro y fuera del aula.

Por otro lado, los docentes están buscan educación de posgrado tanto en niveles de maestría y doctorado, para lo cual tienen que emprender un trabajo de investigación razonable y escribir un trabajo de grado y/o tesis. Lo que se espera de estos programas, es desarrollar al docente como un investigador competente, pero algunos se demoran más de lo esperado o en alguno casos, no logran completar su postgrado, debido a la falta de conocimiento y habilidades para conducir investigación: buscar y seleccionar la información utilizando bibliotecas o la web, recopilar y usar herramientas para analizar los datos y, escribir y comunicar la investigación, entre otras.

Algunos autores como Hernández, Prada y Avendaño (2020); Rocas, Granados y Salcedo (2014); Avilés (2012); Terán (2010), Ollarves y Salguero (2009); Aular, Marcano y Moronta (2009), entre otros, investigaron sobre competencias investigativas en docentes tanto en los niveles básicos como en educación superior, coinciden en la necesidad de formar a los estudiantes para desarrollar habilidades de investigación. Por otro lado, están los trabajo de Ortega y Jaik (2010), Jaik (2013), entro otros, sobre medición y escala de evaluación de competencias investigativas 
De acuerdo con los antecedentes planteados, hay investigaciones que permiten tener una visión amplia y profunda de las competencias de investigación tanto en estudiantes como en docentes de todos los niveles investigativos tanto a nivel nacional como internacional. En este barrido de información no se encuentra muchos trabajos relacionados con el diseño y validación de instrumentos para medir las competencias investigativas.

Además, el problema se plantea porque en la actualidad existe una debilidad en torno a práctica de la función investigativa dentro del contexto de la educación básica y media y no se cuenta con un instrumento validado y confiable que permita medir los indicadores o competencias que debe poseer el docente para incorporar la investigación en su práctica pedagógica. La problemática se agudiza, cuando se evidencia que en su mayoría los docentes no poseen las competencias básicas y profesionales, que les permita efectuar investigaciones de manera continua y sistemática (Álvarez, Orozo y Gutierréz, 2011).

En virtud del fenomeno planteado, el propósito del estudio está basado en la necesidad de diseñar y validar una instrumento (escala) para medir las competencias investigativas que poseen los docentes de los niveles de educación básica y media, con el fin de que pueda ser utilizado en cualquier contexto colombiano.

Con el diseño de este instrumento, se podrá estudiar y analizar cuál es el impacto de las competencias investigativas del docente en los procesos de enseñanza y aprendizaje en estudiantes de básica. Dentro de esto, se espera que la información obtenida al determinar que competencias investigativas que poseen los docentes permita identificar las estrategias pedagógicas que están aplicando, evaluar el impacto que éstas tienen, y así evidenciar la necesidad de construir una propuesta para implementar estrategias pedagógicas que desarrollen las competencias investigativas.

\section{ASPECTOS CONCEPTUALES}

Las competencias investigativas genéricas son aquéllas que, desarrollan los egresados de educación superior, permitiéndoles adquirir capacidades para aplicar conceptos básicos, métodos y técnicas de investigación, y en el caso de los docentes, adecuarlos a las situaciones cotidianas de la vida escolar (Muñoz, Quintero y Munévar, 2001). De igual forma son "el conjunto de conocimientos, actitudes, habilidades y destrezas necesarias para llevar a cabo la elaboración de un trabajo de investigación" (Jaik, 2013, p. 36), por parte de cualquier profesional.

Por otra parte, para los profesionales de la educación, las competencias en investigación se refieren a los requerimientos fundamentales en el área investigativa de los profesionales en educación, adquiridas durante su formación básica (Benavides, 2003). Ademas, las competencias investigativas específicas las desarrolla el docente, en un área específica de un puesto de trabajo, con dominio conceptual y metodológico que le permiten lograr resultados definidos en el proceso investigativo de su labor (Hurtado, 2000).

Las competencias investigativas del docente, son las capacidades y actitudes del docente de someter a crítica lo que hace, cómo lo hace y cómo hacerlo mejor, con dominio de la lógica para resolver cualquier problema; es decir, en una reelaboración del saber, lo cual implica un continuo reflexión-acción en su práctica cotidiana, un despliegue de su capacidad para discernir los modos más apropiados de mejorar la educación, dándole sentido a todas las actividades que ejecuta, mediante el desarrollo de procesos que engloben la construcción de conocimientos encaminados a lograr aprendizajes de calidad y pertinentes 
en los diferentes actores que intervienen en la escuela y en la comunidad (Hernández, Prada y Ramírez, 2019).

Según Muñoz, Quintero y Munévar (2001), las competencias investigativas son aquellas necesarias para que los educadores logren interpretar, argumentar, proponer alternativas, preguntar y escribir a partir de la experiencia pedagógica de acuerdo con la problemática que caracteriza el aula y la escuela. Son una capacidad crítica que debe poseer el docente, para lograr un desempeño académico, eficiente, convirtiendo el quehacer educativo en objeto de estudio y acciones creativas, haciendo de la escuela un espacio de intercambio y confrontación de ideas (Hernández, Prada y Ramírez, 2019). En consecuencia, el docente debe desarrollar habilidades para aplicar los conceptos básicos, los métodos y las técnicas de investigación educativa en las situaciones cotidianas de la vida escolar, tomando en cuenta las características del contexto, y la cultura escolar.

\section{ASPECTOS TÉCNICOS CONSIDERADOS EN LA CONSTRUCCIÓN Y EVALUACIÓN DE ESCALAS DE MEDICIÓN}

El proceso de diseño de escalas. Diseñar y validar escalas sigue todo un proceso, si se quiere secuencial, aunque requiere de una revisión continua, varios autores ilustran este proceso con fines prácticos y didácticos. Blanco, Urosa y Morales (2003) muestran el procedimiento seguido para la construcción de la escala de actitudes, se puede resumir en los siguientes pasos en orden más lógico, que estrictamente cronológico:1. Definir la actitud que se desea medir.; 2. Redactar los ítems ;3. Determinar el modo de respuesta y el número de respuestas adicionales; 4 . Preparar la clave de corrección ;5. Preparar preguntas o instrumentos adicionales en previsión de los estudios para comprobar la validez; 6 . Analizar los ítems: ver si discriminan y si se pueden considerar indicadores del mismo rasgo; 7 . Obtener datos de una muestra ;8. Comprobar la fiabilidad general de la escala;9. Seleccionar los ítems definitivos y 10. Comprobar la validez y otros análisis posteriores.

El juicio de Expertos. La concordancia en el acuerdo en la opinión de los jueces como un indicador de la validez de contenido, se verifica usando pruebas No paramétricas. Si se trata de dos variables con opciones dicotómicas es común el índice Kappa. En el caso de valoraciones que se aprecian como variables ordinales se lleva a cabo la prueba estadística denominada Coeficiente de Concordancia de Kendall ( $W$ de Kendall) para determinar la validez de contenido. Esta prueba en determinar el grado de acuerdo entre varios jueces. Dicha prueba posibilita decidir el nivel de concordancia entre los expertos, cuyo valor oscila entre 0 y 1 . El valor 1 significa una concordancia de acuerdo total y el valor de 0 un desacuerdo total. La fórmula es la siguiente:

$$
W=\frac{S}{\frac{1}{12} K^{2}\left(N^{3}-N\right)-K \sum L i}
$$

Dónde: $W=$ coeficiente de concordancia de Kendall, $S=$ suma de los cuadrados de las diferencias observadas con respecto a un promedio, $N=$ tamaño de la muestra, $K=$ número de variables incluidas y $L i=$ sumatoria de las ligas o empates entre los rangos.

Para determinar la concordancia se realiza por categoría a evaluar, de esta manera se compara el grado de correlación en cada una de ellas, para lo cual se plantea el siguiente sistema de hipótesis, a un nivel de significancia del $5 \%$ :

$\mathrm{H}_{0}$ : No concordancia entre las valoraciones emitidas por los expertos.

$H_{i}$ : Existe concordancia entre las valoraciones emitidas por los expertos. 
El criterio para rechazar la hipótesis nula (Ho) es el siguiente: si el valor de significancia calculado o teórico es mayor a $5 \%(p>0.05)$ significa que no hay concordancia o similitud entre las valoraciones emitidas por los expertos, de lo contrario, se rechaza la hipótesis nula $(p$ $<0.05$ ). A partir de esto realizar la modificación de la redacción de los ítems a partir de las sugerencias de los expertos.

Análisis de la consistencia interna. En lo relativo a la fiabilidad de la escala de medida, se considera la media ponderada de las correlaciones entre las variables (o ítems) que forman parte de la escala, también llamado Alfa de Cronbach ( $\alpha$ ) (Palella y Martins, 2006). El cálculo se realizó con la siguiente fórmula:

$$
\alpha_{e s t}=\frac{k p}{1+p(k-1)}
$$

Dónde: $k$ es el número de ítems, $p$ es el promedio de las correlaciones lineales entre cada uno de los ítems (se tendrán $\frac{[k(k-1)]}{2}$ pares de correlaciones).

En determinados contextos, se considera que valores del alfa superiores a 0,7 ○ 0,8 (dependiendo de la fuente) son suficientes para garantizar la fiabilidad de la escala. Finalmente, la redacción definitiva de la escala se hace a partir de los resultados anteriores.

\section{Análisis de las propiedades psicométricas.}

Un aspecto central en la construcción de escalas es la validez de constructo. Para determinar este tipo de validez se utiliza un análisis factorial exploratorio para medir la homogeneidad y comunalidad de las categorías y subcategorías (estructura y constructo) que conforman el instrumento (Morales, 2013). Es una técnica de reducción de datos que sirve para encontrar grupos homogéneos de ítems a partir de un conjunto numeroso de ellos. Estas asociaciones se forman con las variables que correlacionan mucho entre sí y procurando, inicialmente, que unos grupos sean independientes de otros. En el caso que nos atañe, se efectuó el análisis factorial exploratorio por categorías del estudio, lo que permite observar la relación entre ellas (Morales, 2011).

Para ello se recurrirá al método de componentes principales (ACP), el cual tiene como objetivo transformar un conjunto de variables originales, en un nuevo conjunto de variables ( $\sin$ perder información), combinación lineal de las originales, denominadas componentes principales (factores). El ACP trata de hallar estos componentes o factores, los cuales se caracterizan por estar incorrelacionadas entre sí. En este sentido, para aplicar el análisis factorial se debe tomar una serie de consideraciones con el fin de garantizar que los datos se ajustan o no a un modelo de análisis factorial, para ello los datos obtenidos de la versión definitiva de la escala fueron al test de KMO y esfericidad de Bartlett. La medida de adecuación muestral KMO propuesta por Kaiser, Meyer y Olkin aconsejan que si $\mathrm{KMO} \geq 0.75$ la idea de realizar un análisis factorial es buena, si $0.5 \leq \mathrm{KMO}<0.75$ es aceptable, y si es menor que 0.5 es inaceptable. Por su parte, la prueba de esfericidad de Bartlett contrasta la hipótesis nula de que la matriz de correlaciones es la identidad, y si eso es así, las intercorrelaciones entre las variables es cero, es decir, el modelo es significativo cuando el $p$-valor (o significancia) es menor al $5 \%(p<0,05)$.

\section{MÉTODO}

Naturaleza del Estudio. El estudio es de naturaleza descriptiva, pues a partir de un conjunto de datos relacionados con el conjunto de características de un grupo de personas se busca identificar los aspectos de interés en el grupo analizado, en función de los objetivos y variables de interés del estudio relacionadas con las competencias de investigación de docentes de educación básica en servicio. 
El estudio se encuentra dentro del conjunto de investigaciones denominadas psicométricas, pues en esencia, sigue el proceso para la validación y construcción de escalas de medición en el que se busca destacar las propiedades métricas del instrumento resaltando el proceso de validez, confiabilidad y sensibilidad cultural de la escala de acuerdo con los estándares internacionales. Por la forma dado que se recoge la información, el trabajo puede catalogarse como un estudio de campo pues los datos se recogieron in situ y fueron suministrados por los propios participantes.

Muestra. La escala se aplicó a un total de 32 sujetos con el fin de proceder a realizar la prueba piloto que respondían al perfil de la investigación. En la Tabla 1 se resumen las características de este grupo considerado. El 56,3\% son mujeres y de ellas sólo el $37,5 \%$ tiene edades entre 46 y 60 años. Edad promedio del grupo es de 49,5 años, con una desviación típica de 10,46 años.

Tabla 1. Características de la muestra piloto

\begin{tabular}{|c|l|c|c|c|c|c|c|}
\hline & & \multicolumn{6}{c|}{ Género } \\
\hline & \multicolumn{2}{|c|}{ Masculino } & \multicolumn{2}{c|}{ Femenino } & \multicolumn{2}{c|}{ Total } & \\
\hline \multirow{3}{*}{ Edad } & \multicolumn{1}{|c|}{$\mathrm{f}$} & $\%$ & $\mathrm{~F}$ & $\%$ & $\mathrm{f}$ & $\%$ & \\
\cline { 2 - 9 } & Entre 18 y 30 años & 2 & $6.3 \%$ & 0 & $0.0 \%$ & 2 & $6.3 \%$ \\
\cline { 2 - 8 } & Entre 31 y 45 años & 1 & $3.1 \%$ & 5 & $15.6 \%$ & 6 & $18.8 \%$ \\
\cline { 2 - 8 } & Entre 46 y 60 años & 8 & $25.0 \%$ & 12 & $37.5 \%$ & 20 & $62.5 \%$ \\
\cline { 2 - 8 } & 61 años o más & 3 & $9.4 \%$ & 1 & $3.1 \%$ & 4 & $12.5 \%$ \\
\cline { 2 - 8 } & Total & 14 & $43.8 \%$ & 18 & $56.3 \%$ & 32 & $100.0 \%$ \\
\hline
\end{tabular}

Aproximadamente el $29 \%$ indica haber participado dentro de un proyecto de investigación, aunque sólo el 9\% admitió participar en proyectos financiados. El $76 \%$ posee estudios de especialización y sólo el $6 \%$ de Maestría.

Instrumento. Se construyó un instrumento para medir las competencias investigativas que requieren los docentes de educación básica y media utilizando una escala tipo Likert con cinco (5)alternativas de respuestas: "nada competente" (1), "poco competente" (2), "competente" (3), "muy competente" (4), y "totalmente competente" agrupa los ítems en función de tres dimensiones básicas, constituidas como subescalas. La primera está relacionada con la identificación y organización de la información con un total de 9 ítems. La segunda compuesta por 13 ítems que hacen referencia a la generación científica del conocimiento. Finalmente, la tercera escala está conformada por 9 ítems que se refiere a las competencias relacionadas con la divulgación del conocimiento (Tabla 2). 
Tabla 2. Dimensiones e Ítems del instrumento de competencias investigativas.

\begin{tabular}{|c|c|c|}
\hline Escala 1 Identificación & Escala 2 Generación & Escala 3 Divulgación \\
\hline $\begin{array}{l}\text { Item1. Identifico fuentes de } \\
\text { información que facilitan los } \\
\text { procesos de investigación en el } \\
\text { aula }\end{array}$ & $\begin{array}{l}\text { Item10. Reconozco en la } \\
\text { realidad escolar situaciones } \\
\text { o problemas que pueden } \\
\text { ser objeto relevante de una } \\
\text { investigación educativa } \\
\end{array}$ & $\begin{array}{l}\text { Item23. Conozco los distintos } \\
\text { escenarios (reuniones } \\
\text { científicas y publicaciones) } \\
\text { para la divulgación de la } \\
\text { investigación }\end{array}$ \\
\hline $\begin{array}{l}\text { Item2. Identifico bases de } \\
\text { datos científicas y académicas } \\
\text { para soportar mis procesos de } \\
\text { investigación relacionados con la } \\
\text { práctica pedagógica }\end{array}$ & $\begin{array}{l}\text { Item11. Tengo la capacidad } \\
\text { para formular adecuadamente } \\
\text { como problema de investigación } \\
\text { una situación de la realidad } \\
\text { educativa }\end{array}$ & $\begin{array}{l}\text { Item24. Distingo la necesidad } \\
\text { de adecuar el formato de los } \\
\text { resultados de la investigación } \\
\text { al espacio en el que se } \\
\text { presentan (blogs, artículos } \\
\text { científicos, ponencias y redes } \\
\text { sociales) }\end{array}$ \\
\hline $\begin{array}{l}\text { Item3. Ubico con facilidad } \\
\text { información valida y confiable } \\
\text { disponible en internet }\end{array}$ & $\begin{array}{l}\text { Item12. Tengo claro que toda } \\
\text { investigación debe realizar una } \\
\text { aportación original y relevante a } \\
\text { las Ciencias de la Educación }\end{array}$ & $\begin{array}{l}\text { Item25. Participo en eventos } \\
\text { (charlas, seminarios, } \\
\text { congresos) con el fin de } \\
\text { divulgar los resultados de mis } \\
\text { investigaciones }\end{array}$ \\
\hline $\begin{array}{l}\text { Item4. Utilizo la información } \\
\text { disponible en Internet con una } \\
\text { actitud crítica y reflexiva }\end{array}$ & $\begin{array}{l}\text { Item13. Puedo formular } \\
\text { en términos de hipótesis o } \\
\text { preguntas de investigación un } \\
\text { problema científico }\end{array}$ & $\begin{array}{l}\text { Item26. Reconozco la } \\
\text { importancia de Normas } \\
\text { estandarizadas para las } \\
\text { publicaciones científicas }\end{array}$ \\
\hline $\begin{array}{l}\text { Item5. Utilizo plataformas } \\
\text { especializadas (Repositorios, } \\
\text { bases de datos, software } \\
\text { científico) para sustentar la } \\
\text { investigación en el aula. }\end{array}$ & $\begin{array}{l}\text { Item14. Sé fundamentar ideas } \\
\text { de otros autores para construir } \\
\text { un argumento }\end{array}$ & $\begin{array}{l}\text { Item27. Tengo capacidad } \\
\text { para componer y redactar un } \\
\text { informe de investigación en } \\
\text { forma adecuada }\end{array}$ \\
\hline $\begin{array}{l}\text { Item6. Ordeno los resultados } \\
\text { de la búsqueda de acuerdo con } \\
\text { el interés de la investigación } \\
\text { pedagógica }\end{array}$ & $\begin{array}{l}\text { Item15. Puedo formular en } \\
\text { términos claros y precisos el } \\
\text { propósito de una investigación } \\
\text { pedagógica }\end{array}$ & $\begin{array}{l}\text { Item28. Participo en } \\
\text { redes, basadas en el uso } \\
\text { de tecnologías, para la } \\
\text { construcción colectiva de } \\
\text { conocimiento pedagógico. }\end{array}$ \\
\hline $\begin{array}{l}\text { Item7. Selecciono las fuentes } \\
\text { de acuerdo con su relevancia y } \\
\text { afinidad para el estudio }\end{array}$ & $\begin{array}{l}\text { Item16. Distingo entre las } \\
\text { metodologías de investigación } \\
\text { la más adecuada a un problema } \\
\text { determinado }\end{array}$ & $\begin{array}{l}\text { Item29. Participo en } \\
\text { comunidades virtuales, para } \\
\text { la construcción colectiva de } \\
\text { conocimiento pedagógico con } \\
\text { el apoyo de TIC. }\end{array}$ \\
\hline $\begin{array}{l}\text { Item8. Estoy en capacidad } \\
\text { de exponer sintética y } \\
\text { ordenadamente el conocimiento } \\
\text { previamente publicado } \\
\text { relacionado con la investigación } \\
\text { pedagógica }\end{array}$ & \begin{tabular}{|l|} 
Item17. Selecciono los \\
participantes del estudio a través \\
de procedimientos cónsonos con \\
las metodologías empleadas
\end{tabular} & $\begin{array}{l}\text { Item30. Participo en } \\
\text { proyectos colaborativos, a } \\
\text { través del uso de las TIC, } \\
\text { para la construcción colectiva } \\
\text { de conocimiento pedagógico. }\end{array}$ \\
\hline
\end{tabular}




\begin{tabular}{|l|l|l|}
\hline $\begin{array}{l}\text { Item9. Extraigo las ideas } \\
\text { originales de otros autores y las } \\
\text { incorporo apropiadamente para } \\
\text { soportar mis argumentos }\end{array}$ & $\begin{array}{l}\text { Item18. Reconozco el tipo de } \\
\text { instrumento de recolección de } \\
\text { datos adecuado a la naturaleza y y } \\
\text { propósito de la investigación }\end{array}$ & $\begin{array}{l}\text { Item31. Selecciono } \\
\text { apropiadamente el medio } \\
\text { de divulgación de una } \\
\text { investigación de acuerdo } \\
\text { con la pertinencia de los } \\
\text { resultados obtenidos a la } \\
\text { línea editorial }\end{array}$ \\
\hline & $\begin{array}{l}\text { Item19. Conozco herramientas } \\
\text { básicas de análisis estadístico } \\
\text { para el tratamiento de los } \\
\text { resultados obtenidos en } \\
\text { investigaciones }\end{array}$ & \\
\hline & $\begin{array}{l}\text { Item20. Tengo capacidad para } \\
\text { elaborar tablas o gráficos que } \\
\text { resumen el producto de mis } \\
\text { investigaciones }\end{array}$ & \\
\hline & $\begin{array}{l}\text { Item21. Conozco métodos para } \\
\text { analizar datos de naturaleza no } \\
\text { numéricas }\end{array}$ & \\
\hline & $\begin{array}{l}\text { Item22. Estoy en capacidad } \\
\text { de contrastar los resultados } \\
\text { obtenidos con los de otras } \\
\text { investigaciones y posiciones } \\
\text { teóricas }\end{array}$ & \\
\hline &
\end{tabular}

Diseño del estudio. El presente estudio pretende abordar el proceso de Construcción de una escala de Medición. Para efectos de este estudio se sigue el modelo sugerido por Zapata y Canet, (2008). El estudio comprende los 8 primeros pasos, dejando el estudio de la validez del constructo para una futura investigación. Los pasos, según relacionan estos autores, comprenden:
1. Especificación del Constructo

2. Definición de las Dimensiones

3. Selección de los Ítems y la Técnica de Escalamiento

4. Diseño de la Población y la Muestra

5. Prueba Piloto

6. Ajuste de la Escala

7. Aplicación del Cuestionario

8. Ajuste Final de la Escala

9. Fiabilidad y Validez del Constructo

10. Diseño del Path Diagram 


\section{RESULTADOS}

Valoraciones realizadas por los jueces. En la Tabla 3 se exponen las valoraciones que realizaron los jueces a cada según las tres escalas, considerando para cada ítem los criterios de claridad tendencia y tendenciosidad en una escala de 1 a 3 puntos.

Tabla 3. Resultados del Juicio de Expertos

\begin{tabular}{|c|c|c|c|c|c|}
\hline \multicolumn{2}{|c|}{ Escala 1 Identificación } & \multicolumn{2}{c|}{ Escala 2 Generación } & \multicolumn{2}{c|}{ Escala 3 Divulgación } \\
\hline & $\begin{array}{c}\text { Rango } \\
\text { promedio }\end{array}$ & & $\begin{array}{c}\text { Rango } \\
\text { promedio }\end{array}$ & $\begin{array}{c}\text { Rango } \\
\text { promedio }\end{array}$ \\
\hline item1 & 6.00 & item10 & 9.33 & item23 & 6.33 \\
\hline item2 & $3.00^{*}$ & item11 & 9.33 & item24 & $1.33^{*}$ \\
\hline item3 & 6.00 & item12 & $2.83^{*}$ & item25 & 6.33 \\
\hline item4 & 6.00 & item13 & 7.17 & item26 & 6.33 \\
\hline item5 & 6.00 & item14 & 9.33 & item27 & 6.33 \\
\hline item6 & 6.00 & item15 & 9.33 & item28 & 4.67 \\
\hline item7 & 6.00 & item16 & 7.17 & item29 & 6.33 \\
\hline item8 & $3.00^{*}$ & item17 & 2.83 & item30 & 6.33 \\
\hline item9 & $3.00^{*}$ & item18 & 7.17 & item31 & 4.67 \\
\hline & & item19 & 7.17 & item32 & 6.33 \\
\hline & & item20 & 9.33 & & \\
\hline & & item21 & 2.83 & & \\
\hline & & item22 & 7.17 & & \\
\hline
\end{tabular}

* Items con sugerencia de Revisión

La Figura 1 permite observar los rangos promedios por indicador entre jueces en las evaluaciones de las tres escalas. Se puede apreciar que los rangos son variables y oscilan entre 1,33 y 9,33 . Para las escalas 1 y 3 , relacionadas con la identificación de información y con la divulgación del conocimiento científico, los rangos promedios se observan más bajos en comparación con la escala 2 de Generación del conocimiento científico, especialmente con los ítems 10, 11 y 20 referidos a la identificación del problemas de investigación, su formulación y la presentación de resultados mediante tablas o gráficos, consideradas por los jueces como competencias claves para la generación del conocimiento científico. 


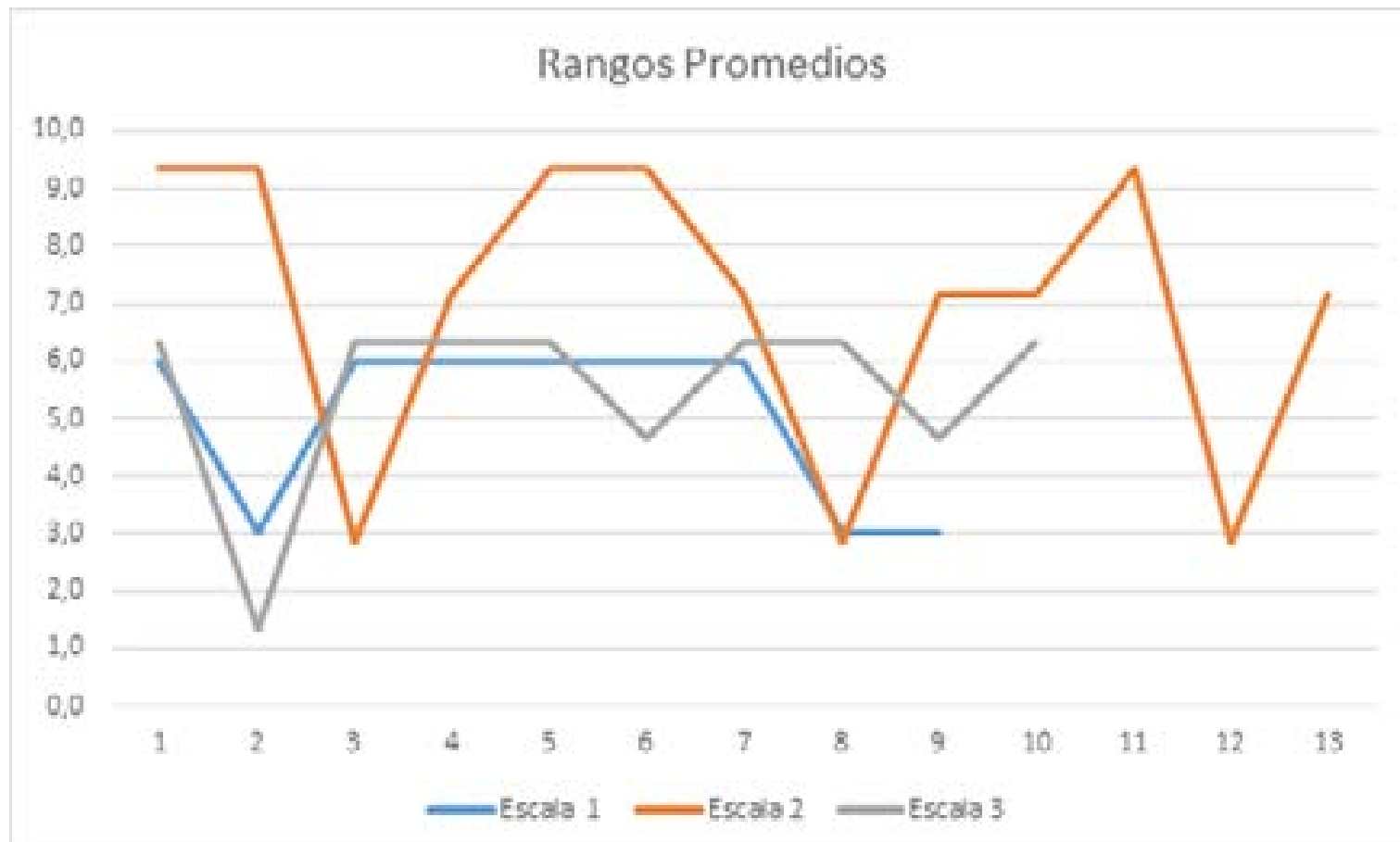

Figura 1. Rangos promedios de los jueces en las escalas de identificación (1), generación (2) y divulgación (3) del conocimiento científico.

El análisis de los valores promedios alcanzados en cada uno de los ítems del instrumento usado por los expertos para expresar su juicio permite también explicar con más precisión en qué consistió la coincidencia alcanzada, en relación con los tres criterios claridad del ítem, congruencia con la escala empleada y ausencia de tendenciosidad o ítems con respuestas sugerentes.

En la dimensión de identificación de información los ítems con rangos promedios menos valorados son los ítems 2, 8 y 9, que hacen referencia al manejo de bases de datos académicas, síntesis y ordenamiento de información previamente publicada y la referencia a otros autores.

Con respecto a la divulgación de la información la mayoría de los ítems tienen rangos promedios próximos a 5 o superiores a este valor con excepción del ítem 24 que presente el rango promedio más bajo del conjunto y que hace referencia a la habilidad para distinguir entre varios formatos para publicar información o productos de investigaciones, esto es: blogs, artículos científicos, ponencias y redes sociales.

Con el fin de valorar la opinión de los jueces, al conjunto de datos proporcionado por los expertos se le aplicó la prueba estadística no paramétrica denominada coeficiente de concordancia de $W$ de Kendall. Según Pardo y Ruiz (2011), el coeficiente de concordancia $W$ sirve para estudiar la concordancia entre un conjunto de rangos, por ejemplo, cuando $n$ expertos evalúan según una característica, como es el caso de la evaluación de los indicadores del blog diseñado. Existe concordancia cuando los expertos valoran del mismo modo (Ver tabla 4). 
Tabla 4. Valores de $W$ de Kendall

\begin{tabular}{|l|c|c|c|}
\hline & Escala 1 & Escala 2 & Escala 3 \\
\hline $\mathrm{N}$ & 3 & 3 & 3 \\
\hline W de Kendall & 0,667 & 0,643 & 0,691 \\
\hline Chi-cuadrado & 16,000 & 23,158 & 18,65 \\
\hline GI & 8 & 12 & 9 \\
\hline Sig. asintót. & 0,042 & 0,026 & 0,028 \\
\hline
\end{tabular}

Al realizar la evaluación, para la Escala 1 se obtuvo un valor de $W$ de Kendall equivalente a 0,66 de acuerdo con los datos expuestos en la tabla 4, además el valor del nivel crítico (Sig. Asintótica) es menor de 0,05, en consecuencia, se puede rechazar la hipótesis de concordancia nula y concluir que entre las puntuaciones estudiadas de los tres expertos existe concordancia significativa. Similarmente para las escalas 2 y 3 , los valores de $W$ de Kendall se ubicaron en 0,64 y 0,69 respectivamente, con $p$-valores asociado de $p=0,026$ y $p=$ 0,028 ambos $<0,05$ verificando de este modo la hipótesis de concordancia.

Prueba piloto y análisis de ítems. El proceso de aplicación de la prueba piloto consistió en administrar el instrumento a un número reducido de docentes con el fin de valorar sus propiedades psicométricas. Una vez registrados los ítems de la prueba piloto se procedió a calcular el Alfa de Cronbach y la correlación ítem total. El valor de Cronbach para la escala global alcanzó el 0,84 y la correlación ítem total (Ver Tabla III) permitió valorar la calidad de cada ítem individual, en función de su índice de discriminación y la correlación con el conjunto.

Para contrastar dicho coeficiente, se realizó un análisis individual de cada ítem, centrado en la correlación ítem-total, que permitió valorar la relación de cada ítem con el total; este estadístico considera que para valores mayores a 0,30 los ítems discriminan bien, según Muñiz (2005) caracteriza a un ítem que justifica su presencia en la escala. La Figura 2 muestra las correlaciones ítem-total de los ítems analizados. La Tabla 5 muestra el conjunto de ítems que, en general, tienen un buen comportamiento en la medición de los constructos analizados, los valores de la correlación ítem en total oscilan entre 0,30 y 0,80 , y su valor medio alcanza 0,57 .

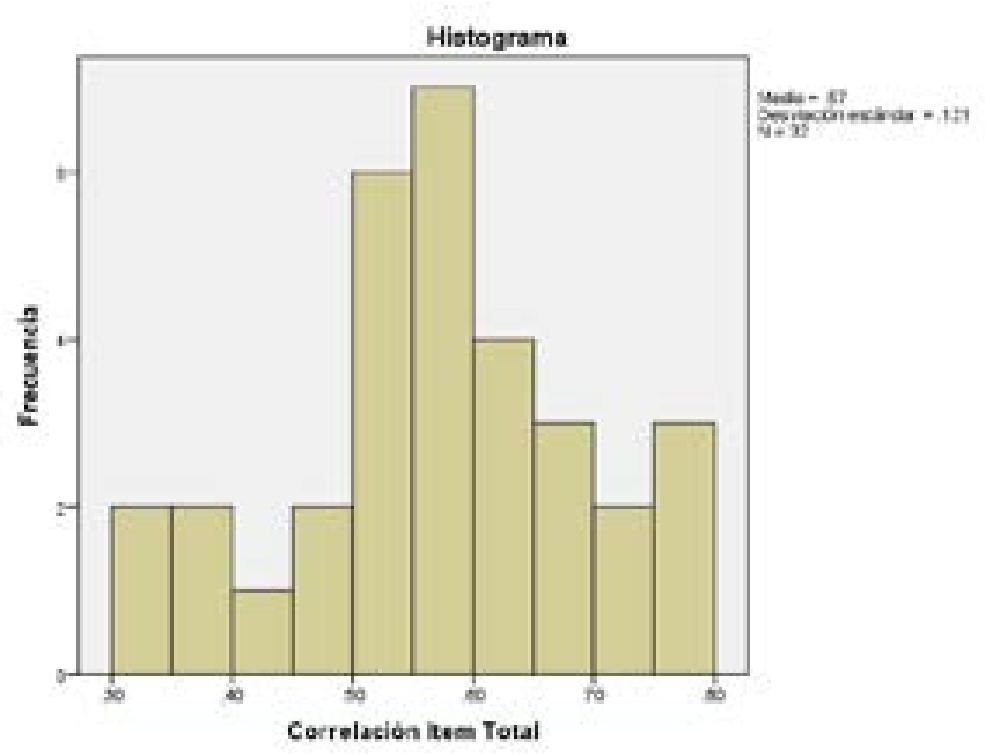

Figura 2. Correlación ítem-total de los ítems que conforman el cuestionario de la prueba piloto. 
Tabla 5. Estadísticos total-elemento.

\begin{tabular}{|c|c|c|c|c|}
\hline & $\begin{array}{c}\text { Media de } \\
\text { escala si el } \\
\text { elemento se } \\
\text { ha suprimido }\end{array}$ & $\begin{array}{l}\text { Varianza de } \\
\text { escala si el } \\
\text { elemento se } \\
\text { ha suprimido }\end{array}$ & $\begin{array}{l}\text { Correlación } \\
\text { total de } \\
\text { elementos } \\
\text { corregida }\end{array}$ & $\begin{array}{c}\text { Alfa de } \\
\text { Cronbach si } \\
\text { el elemento } \\
\text { se ha } \\
\text { suprimido }\end{array}$ \\
\hline item1 & 112.48 & 249.875 & .322 & .941 \\
\hline item2 & 112.67 & 251.308 & .362 & .940 \\
\hline item3 & 112.30 & 246.986 & .523 & .938 \\
\hline item4 & 112.11 & 245.179 & .602 & .938 \\
\hline item5 & 112.70 & 244.217 & .577 & .938 \\
\hline item6 & 112.59 & 242.866 & .647 & .937 \\
\hline item7 & 112.41 & 244.866 & .593 & .938 \\
\hline item8 & 112.22 & 247.256 & .600 & .938 \\
\hline item9 & 112.70 & 243.140 & .476 & .939 \\
\hline item10 & 112.33 & 244.846 & .734 & .937 \\
\hline item11 & 112.44 & 246.333 & .555 & .938 \\
\hline item12 & 112.30 & 247.524 & .537 & .938 \\
\hline item13 & 112.56 & 244.103 & .647 & .937 \\
\hline item14 & 112.48 & 243.644 & .694 & .937 \\
\hline item15 & 112.41 & 241.789 & .763 & .936 \\
\hline item16 & 112.48 & 243.259 & .711 & .937 \\
\hline item17 & 112.81 & 248.695 & .532 & .938 \\
\hline item18 & 112.74 & 247.199 & .560 & .938 \\
\hline item19 & 112.81 & 243.695 & .662 & .937 \\
\hline item20 & 112.70 & 246.601 & .551 & .938 \\
\hline item21 & 112.85 & 243.208 & .528 & .938 \\
\hline item22 & 112.81 & 242.387 & .674 & .937 \\
\hline item23 & 112.48 & 241.028 & .757 & .936 \\
\hline item24 & 112.89 & 243.487 & .568 & .938 \\
\hline item25 & 112.93 & 239.225 & .761 & .936 \\
\hline item26 & 113.11 & 243.872 & .451 & .940 \\
\hline item27 & 113.00 & 245.077 & .549 & .938 \\
\hline item28 & 112.67 & 248.615 & .553 & .938 \\
\hline item29 & 113.30 & 248.832 & .378 & .940 \\
\hline item30 & 113.41 & 250.866 & .336 & .940 \\
\hline item31 & 113.30 & 246.063 & .433 & .940 \\
\hline item32 & 113.33 & 245.000 & .524 & .938 \\
\hline
\end{tabular}


Confiabilidad de las subescalas. La evaluación del comportamiento individual de cada escala es muy positiva. Para la escala 1 relacionada con la identificación y búsqueda de información el estadístico Alfa de Cronbach, y arrojó un valor de 0,89 , similarmente la escala 2 de generación de conocimiento alcanzó un valor de Alfa de 0,91 y la escala 3 de divulgación de conocimiento obtuvo un valor de 0,89 , estos valores se ubican en el rango de muy alta confiabilidad, de acuerdo con lo indicado por Pardo y Ruiz (2002) y Bolívar (1995). En consecuencia, se puede decir que la escala diseñada goza de credibilidad y consistencia interna y se puede tomar como referente para futuras aplicaciones.

\section{CONCLUSIONES}

Para construir un instrumento que mida las competencias investigativas del docente de educación básica y media se trabajó con las siguientes dimensiones: identificación y organización de la información (9 ítems), generación científica del conocimiento (13 ítems), y divulgación del conocimiento científico (9 ítems) para un total de 31 ítems, además de unas preguntas para analizar el perfil de los docentes objeto de estudio.

Con el proceso presentado para el diseño y validación de un instrumento para la medición de las competencias investigativas en los docentes de educación básica y media, gira en torno al desarrollo de un cuestionario, donde ha sido necesario tomar en cuenta un sistema de fundamentos metodológicos. En este sentido, el instrumento y sus escalas serán sometidos a un riguroso proceso de validación y confiabilidad, para lo cual se utilizará el coeficiente de concordancia de Kendall, el Alpha de Cronbach y el Análisis Factorial Exploratorio.

El análisis de ítems, principalmente el juicio de expertos sugirió la revisión parcial en cuanto a algunos ítems, específicamente relacionados con algunos aspectos técnicos como el iteme2 (manejo de bases de datos académicas) y el ítem 24 (identificación de distintos formatos de publicación), sin embargo en el análisis de la confiabilidad de cada sub escala estos ítems resultaron con una correlación ítem-total superior a 0,30 , hecho que ratifica la consistencia de los ítems y en consecuencia de las sub escalas y de la medida global.

Aunque los resultados del proceso de validación y confiabilidad fueron satisfactorios, las actividades de investigación derivadas de este trabajo están enfocadas hacia el análisis de la validez de constructo con una muestra de sujetos más amplia y a través de técnicas de análisis multivariante como el análisis factorial exploratorio y a partir de métodos confirmatorios como los modelos de ecuaciones estructurales.

Sobre la aplicación del cuestionario a los docentes sujetos de estudio o prueba piloto se obtuvo que aun cuando éstos tienen conocimiento sobre aspectos relacionados con la investigación, la mayoría no la aplica en su práctica de aula o profesional, probablemente debido al escaso manejo o poca practica de saber hacer de un proyecto de investigación, así como debilidades en la divulgación del conocimiento, lo cual evidencia dificultades en las competencias investigativas de los docentes.

\section{REFERENCIAS BIBLIOGRÁFICAS}

Álvarez, V, Orozco, O y Gutiérrez, A. (2011.). La formación de competencias investigativas profesionales, una mirada desde las ciencias pedagógicas. Cuadernos de Educación y Desarrollo, $3(24)$.

Aular, J. A., Marcano, N., y Moronta, M. (2009). Competencias investigativas del docente de educación básica. Laurus, 15(30), 138-165. 
Avilés. A. (2012). Diseño de un Modelo de Gestión del Conocimiento para desarrollar Competencias Investigativas en la educación media y superior en Guayaquil. Ciencia y Tecnología. Revista de Investigación Científica, 1(3).

Benavides, O. (2003). Competencias y Competitividad. Bogotá: Editorial McGraw Hill.

Blanco, A., Sanz, B., y Vallejo, P. (2003). Construcciones de escalas de actitudes tipo Likert. Madrid: Muralla.

Hernández, C., Prada, R. y Ramírez, P. (2019). Competencias TIC e investigativas entre docentes de educación básica. Bogotá: ECOE -Universidad Francisco de Paula Santander.

Hernández-Suárez, C. A., Prada-Núñez, R., y Avendaño-Castro, W. R. (2020). Escala para la valoración de las competencias de producción científica en docentes de Educación Superior. Espacios, 41(44), 113-129. https://www.revistaespacios. com/a20v41n44/a20v41n44p09.pdf

Hurtado, J. (2000). Retos y alternativas en la formación de investigadores. Caracas: Editorial Sypal.

Jaik, D. (2013). Competencias investigativas: Una mirada a la Educación Superior. México: Instituto Politécnico Nacional CIIDIR Unidad Durango.

Morales, P. (2008). El análisis factorial en la construcción e interpretación de tests, escalas y cuestionarios. Madrid: Ed. Universidad Pontificia Comillas.

Morales, P. (2011). Guía para construir cuestionarios y escalas de actitudes. Guatemala: Universidad Rafael Landivar.
Muñoz, J. (2005). La validez desde una óptica psicométrica. Acta Comportamentalia, 13, 9-20.

Muñoz, J.; Quintero, J. y Munevar, R. (2001) Competencias Investigativas para profesionales que forman y enseñan. Bogotá. Editorial Magisterio.

Ollarves, Y. C., y Salguero, L. A. (2009). Una propuesta de competencias investigativas para los docentes universitarios. Laurus, 15(30), 118137.

Ortega, R. y Jaik, D. (2010). Escala de evaluación de competencias investigativas. Revista Electrónica Praxis Investigativa ReDIE, 2(3), 72-75.

Palella, S. y Martins, F. (2006). Metodología de la investigación cuantitativa. Caracas: FEDUPEL.

Pardo, A. y Ruiz, M. (2002). SPSS 11: Guía para análisis de datos. España: Mcgraw-Hill Interamericana.

Roca, M., Granados, A., y Salcedo, D. (2014). Competencias Investigativas en Docentes del Departamento de Ciencias Básicas en la Universidad de la Costa. Psicoespacios, 8(13), 71-99.

Terán, G. (2010). Formación y Gestión de Desempeño del Docente-Investigador en la Educación Superior: Modelo Teórico Basado en Competencias. EIDOS, 3, 53-59.

Zapata, J., y Canet, M. (2008). Propuesta metodológica para la construcción de escalas de medición a partir de una aplicación empírica. Revista Electrónica Actualidades Investigativas en Educación, 8(2), 1-26. 